\title{
Identification of a hereditary system with distributed delay
}

\author{
Arunabha BAGCHI \\ Department of Applied Mathemalics, Twente University of Technolog;; P.O. Box 217, 7500 AE Enschede, The Netherlands
}

Received 7 September 1984

Revised 14 January 1985

We study the identification problem that arises in a linear hereditary system with distributed delay. This involves extimating an infinite-dimensional parameter and we use the method of sieves, proposed by Grenander, to solve this problem.

Key'rords: Hereditary system, Distributed delay, Method of sieves, Consistent estimates.

\section{Introduction}

Although extensive research has been done on the distributed system parameter identification problem (see [1] for a recent survey), relatively little attention has been paid, in the stochastic framework, to the problem of estimating unknown parameters that are infinite dimensional. This is not surprising, as Rozanov pointed out in his classic paper [2] that directly estimating infinite-dimensional parameters by the method of maximum likelihood either may not be possible or may turn out to be not consistent. To circumvent these difficulties, Grenander [3] suggested the method of sieves to estimate parameters in abstract spaces. We apply his idea to a canonical example where one wants to identify the distributed delay function of a linear hereditary system. Recently, Nguyen and Pham [4] applied the method of sieves to the problem of identifying a nonstationary diffusion model.

\section{Problem formulation and estimation procedure}

Let $X_{t},-\infty<t<\infty$, be the stationary solution of a linear time-delayed system with distributed delay:

$$
\mathrm{d} X_{t}=\int_{-b}^{0} \alpha(\theta) X_{t+\theta} \mathrm{d} \theta \mathrm{d} t+\mathrm{d} W_{t}
$$

where, for simplicity, we assume $X$, to be a scalar process and $\left\{W_{t},-\infty<t<\infty\right\}$ is a standard Brownian motion. Itô and Nisio [5] give a sufficient condition for the existence and uniqueness of the stationary solution $X_{t},-\infty<t<\infty$, of (2.1). Assume that $X$, is completely observed in an interval $t_{0} \leqslant t \leqslant T$ and, based on this observation, we want to estimate the function $\alpha(\theta)$ in $[-b, 0]$. We also assume that $a(\cdot) \in L_{2}[-b, 0]$, the space of square-integrable functions on $[-b, 0]$, with $(\cdot, \cdot)$ denoting inner product there. We could also start with the one-sided version of (2.1), i.e. for

$$
X_{t}=\xi(t), \quad-b \leqslant t \leqslant 0,
$$

where $\xi(t)$ is a given element of $L_{2}[-b, 0]$. If the system was stable, the following analysis would go through for the one-sided version of the problem, if we started our identification procedure after the system had reached the steady state. $X_{t}$ is mean-square continuous and has continuous sample paths.

Let $p_{X}$ be the measure induced by $\left\{X_{t}, t_{0} \leqslant t \leqslant T\right\}$ on $C\left[t_{0}, T\right]$, the space of continuous functions on 
$\left[t_{0}, T\right]$, with $p_{W}$ denoting the Wiener measure thereon. From Lipster and Shiryayev [6], we know that $p_{X}$ is absolutely continuous with respect to $p_{w}$ and the log-likelihood functional for the problem is given by

$$
L^{T}(\alpha(\cdot)) \triangleq \frac{\mathrm{d} p_{X}}{\mathrm{~d} p_{W}}(X)=\int_{t_{0}}^{T}\left(\int_{-b}^{0} \alpha(\theta) X_{t+\theta} \mathrm{d} \theta\right) \mathrm{d} X_{t}-\frac{3}{2} \int_{t_{0}}^{T}\left(\int_{-h}^{0} \alpha(\theta) X_{t+\theta} \mathrm{d} \theta\right)^{2} \mathrm{~d} t .
$$

The underlying idea of the method of sieves is that, instead of trying to estimate the function $\alpha(\cdot)$, one estimates a finite-dimensional projection of $\alpha(\cdot)$ of sufficiently high dimension to obtain a good enough estimate of the unknown parameter function. Basically, one constructs an increasing sequence $V_{n}$ of subspaces in $L_{2}[-b, 0]$ of dimension $n_{T}$ such that $U_{n}>_{1} V_{n}$ is dense in $L_{2}[-b, 0]$ and seeks a maximum of $L^{T}(\alpha(\cdot))$ only on the subspace $V_{n}$. Suppose that $\phi_{j}, j=1,2, \ldots$, is a sequence of orthonormal vectors in $L_{2}[-b, 0]$ such that $\left\{\phi_{1}, \phi_{2}, \ldots, \phi_{n_{r}}\right\}$ forms a basis of $V_{n}$ for all $n$. If $\alpha(\cdot) \in V_{n}$, we may write

$$
\alpha(\cdot)=\sum_{j=1}^{n_{r}} \alpha_{j} \phi_{j}(\cdot)
$$

and the log-likelihood functional can be expressed as

$$
\begin{aligned}
L^{T}(\alpha(\cdot)) & =\int_{t_{0}}^{T}\left(\sum_{j=1}^{n_{T}} \alpha_{j} \int_{-b}^{0} \phi_{j}(\theta) X_{t+\theta} \mathrm{d} \theta\right) \mathrm{d} X_{t}-\frac{1}{2} \sum_{j=1}^{n_{T}}\left(\alpha_{j} \int_{-b}^{0} \phi_{j}(\theta) X_{t+\theta} \mathrm{d} \theta\right)^{2} \mathrm{~d} t \\
& =\left(T-t_{0}\right)\left\{b^{(T)^{\prime}} \alpha^{(T)}-\frac{1}{2} \alpha^{(T) '} A^{(T)} \alpha^{(T)}\right\}
\end{aligned}
$$

where $\alpha^{(T)}=\left(\alpha_{1}, \alpha_{2}, \ldots, \alpha_{n_{1}}\right)^{\prime}, b^{(T)}$ is a vector with $j$-th component

$$
b_{j}^{(T)}=\frac{1}{T-t_{0}} \int_{t_{0}}^{T}\left(\int_{-b}^{0} \phi_{j}(\theta) X_{t+\theta} \mathrm{d} \theta\right) \mathrm{d} X_{i}, \quad j=1,2, \ldots, n_{T},
$$

and $A^{(r)}$ is a matrix with $i j$-th component

$$
a_{i j}^{(T)}=\frac{1}{T-t_{0}} \int_{t_{0}}^{T} \int_{-b}^{0} \int_{-b}^{0} \phi_{i}(\theta) X_{t+\theta} X_{t+\theta^{*}} \phi_{j}\left(\theta^{\prime}\right) \mathrm{d} \theta \mathrm{d} \theta^{\prime} \mathrm{d} t, \quad i, j=1,2, \ldots, n_{T} .
$$

The ML (maximum likelihood) estimate of $\alpha(\cdot)$ on $V_{n}$ is then

$$
\hat{\alpha}^{(T)}(\cdot)=\sum_{j=1}^{n_{T}} \hat{\alpha}_{j}^{(T)} \phi_{j}(\cdot)
$$

where $\hat{\alpha}^{(T)}=\left(\hat{\alpha}_{1}^{(T)}, \ldots, \hat{\alpha}_{n}^{(T)}\right)$ is the solution of $A^{(T)} \hat{\alpha}^{(T)}=b^{(T)}$. If $A^{(T)}$ is nonsingular, $\hat{\alpha}^{(T)}=\left[A^{(T)}\right]^{-1} b^{(T)}$. Note that the equation $A^{(T)} \hat{\alpha}^{(T)}=b^{(T)}$ can equivalently be expressed as

$$
\forall h \in V_{n}: \frac{1}{T-t_{0}} \int_{t_{0}}^{r} \int_{-b}^{0} \int_{-b}^{0} h(\theta) X_{t+\theta} X_{t+\theta^{\prime}} \hat{\alpha}^{(r)} \mathrm{d} \theta \mathrm{d} \theta^{\prime} \mathrm{d} t=\frac{1}{T-t_{0}} \int_{t_{0}}^{T}\left(\int_{-b}^{0} h(\theta) X_{t+\theta} \mathrm{d} \theta\right) \mathrm{d} X_{t} .
$$

\section{Consistency of the ML estimate}

In order to study the desired consistency property of our estimate, we introduce another orthonormal basis of $V_{n}$ relative to a different inner product. Let $R(t)$ denote the covariance function of the stationary process $X_{t}$. From [5, p. 52], we know that the process $X$, has a spectral density $S(\lambda)$ given by

$$
S(\lambda)=\frac{1}{\left|\mathrm{i} \lambda-\int_{-b}^{0} \mathrm{e}^{\mathrm{i} \lambda \theta} \alpha(\theta) \mathrm{d} \theta\right|^{2}}
$$

provided $\int_{-b}^{0} \alpha(\theta) \mathrm{d} \theta \neq 0$. Clearly, $S(\lambda)>0$ for all $\lambda$. 
In the space of measurable functions on $[-b, 0]$, we introduce a new inner product

$$
\begin{aligned}
(f, f)_{R} & =\int_{-b}^{0} \int_{-b}^{0} f(\theta)\left(\int_{-\infty}^{\infty} \mathrm{e}^{\mathrm{i} \lambda\left(\theta-\theta^{\prime}\right)} S(\lambda) \mathrm{d} \lambda\right) f\left(\theta^{\prime}\right) \mathrm{d} \theta \mathrm{d} \theta^{\prime} \\
& =\int_{-\infty}^{\infty}\left(\int_{-b}^{0} f(\theta) \mathrm{e}^{i \lambda \theta} \mathrm{d} \theta\right)^{2} S(\lambda) \mathrm{d} \lambda \text { by Fubini's theorem }
\end{aligned}
$$

Then $(f, f)_{R}=0$ implies $\int_{-b}^{0} f(\theta) \mathrm{e}^{\mathrm{i} \lambda \theta} \mathrm{d} \theta=0$ for all $\lambda$, since $\int_{-b}^{0} f(\theta) \mathrm{e}^{\mathrm{i} \lambda \theta} \mathrm{d} \theta$ is a continuous function of $\lambda$ and $S(\lambda)>0$ for all $\lambda$, and then $f(\theta)=0$ a.e.

Furthermore, $R(t)$ being continuous, it is bounded on any compact set and

$$
(f, f)<\infty \Rightarrow(f, f)_{R}<\infty \text {. }
$$

Let $L_{2}^{R}[-b, 0]$ be the completion of $L_{2}[-b, 0]$ with respect to the metric induced by the inner product $(\cdot, \cdot)_{R}$. Consider a sequence $\psi_{1}, \psi_{2}, \ldots$ such that $\psi_{1}, \psi_{2}, \ldots, \psi_{n_{T}}$ form an orthonormal basis of $V_{n}$ for all $n$, relative to the new inner product. Let $\xi_{1}^{(T)}, \hat{\xi}_{2}^{(T)}, \ldots, \hat{\xi}_{n_{T}}^{(T)}$ be the coordinates of $\hat{\alpha}^{(T)}(\cdot)$ in the new basis $\left\{\psi_{1}, \psi_{2}, \ldots, \psi_{n_{r}}\right\}$ of $V_{n}$. From (2.7), it is clear that $\hat{\xi}^{(T)}=\left(\hat{\xi}_{1}^{(T)}, \hat{\xi}_{2}^{(T)}, \ldots, \hat{\xi}_{n_{\tau}}^{(T)}\right)$ is the solution of

$$
F^{(T)} \hat{\xi}^{(T)}=g^{(T)}
$$

where the matrix $F^{(T)}$ and vector $g^{(T)}$ have components

$$
\begin{aligned}
f_{i j}^{(T)} & =\frac{1}{T-t_{0}} \int_{t_{0}}^{T} \int_{-b}^{0} \int_{-b}^{0} \psi_{i}(\theta) X_{t+\theta} X_{t+\theta^{*}} \psi_{j}\left(\theta^{\prime}\right) \mathrm{d} \theta \mathrm{d} \theta^{\prime} \mathrm{d} t, \quad i, j=1,2, \ldots, n_{T} \\
g_{j}^{(T)} & =\frac{1}{T-t_{0}} \int_{t_{0}}^{T}\left(\int_{-b}^{0} \psi_{j}(\theta) X_{t+\theta} \mathrm{d} \theta\right) \mathrm{d} X_{t}, \quad j=1,2, \ldots, n_{T} \\
& =\frac{1}{T-t_{0}} \int_{t_{0}}^{T}\left(\int_{-b}^{0} \psi_{j}(\theta) X_{t+\theta} \mathrm{d} \theta\right)\left(\int_{-b}^{0} \alpha\left(\theta^{\prime}\right) X_{t+\theta^{\prime}} \mathrm{d} \theta\right) \mathrm{d} t+\frac{1}{T-t_{0}} \int_{t_{0}}^{T}\left(\int_{-b}^{0} \psi_{j}(\theta) X_{t+\theta} \mathrm{d} \theta\right) \mathrm{d} W_{i} .
\end{aligned}
$$

Let $\alpha^{(T)}(\cdot)=\sum_{j=1}^{n} \xi_{j} \psi_{j}(\cdot)$ be the orthogonal projection of $\alpha(\cdot)$ onto $V_{n}$ in the sense of the inner product $(\cdot, \cdot)_{R}$. Then the first term on the right-hand side of (3.5) can be written as

$$
\begin{aligned}
& \sum_{k=1}^{n_{T}} f_{j k}^{(T)} \xi_{k}+\frac{1}{T-t_{0}} \int_{t_{0}}^{T}\left(\int_{-b}^{0} \psi_{j}(\theta) X_{t+\theta} \mathrm{d} \theta\right)\left(\int_{-b}^{0}\left[\alpha\left(\theta^{\prime}\right)-\alpha^{(T)}\left(\theta^{\prime}\right)\right] X_{t+\theta^{\prime}} \mathrm{d} \theta\right) \mathrm{d} t \\
& =\sum_{t=1}^{n_{T}} f_{j k}^{(T)} \xi_{k}+\frac{1}{T-t_{0}} \int_{t_{0}}^{T} \int_{-b}^{0} \int_{-b}^{0} \psi_{j}(\theta)\left\{X_{t+\theta} X_{t+\theta^{\prime}}-R\left(\theta-\theta^{\prime}\right)\right\}\left[\alpha\left(\theta^{\prime}\right)-\alpha^{(T)}\left(\theta^{\prime}\right)\right] \mathrm{d} \theta \mathrm{d} \theta^{\prime} \mathrm{d} t
\end{aligned}
$$

since

$$
\int_{-b}^{0} \int_{-b}^{0} \psi_{j}(\theta) R\left(\theta-\theta^{\prime}\right)\left[\alpha\left(\theta^{\prime}\right)-\alpha^{(T)}\left(\theta^{\prime}\right)\right] \mathrm{d} \theta \mathrm{d} \theta^{\prime}=0, \quad j=1,2, \ldots, n_{T}
$$

Therefore, (3.3) can be equivalently written as

$$
F^{(r)}\left(\hat{\xi}^{(T)}-\xi^{(T)}\right)=h^{(T)}
$$

where $\xi^{(T)}$ and $h^{(T)}$ are vectors with components $\xi_{j}, j=1,2, \ldots, n_{T}$, and

$$
\begin{aligned}
h_{j}^{(T)}= & \frac{1}{T-t_{0}} \int_{t_{0}}^{T} \int_{-b}^{0} \int_{-b}^{0} \psi_{j}(\theta) Z_{t}\left(\theta, \theta^{\prime}\right)\left[\alpha\left(\theta^{\prime}\right)-\alpha^{(T)}\left(\theta^{\prime}\right)\right] \mathrm{d} \theta \mathrm{d} \theta^{\prime} \mathrm{d} t \\
& +\frac{1}{T-t_{0}} \int_{t_{0}}^{T}\left(\int_{-b}^{0} \psi_{j}(\theta) X_{t+\theta} \mathrm{d} \theta\right) \mathrm{d} W_{t} \\
= & \frac{1}{T-t_{0}} \int_{t_{0}}^{T} \eta_{j}^{(T)}(t) \mathrm{d} t+\frac{1}{T-t_{0}} \int_{t_{0}}^{T} \bar{\eta}_{j}(t) \mathrm{d} W_{t}
\end{aligned}
$$


Volume 5, Number 5

SYSTEMS \& CONTROL LETTERS

April 1985

where

$$
\begin{aligned}
& Z_{t}\left(\theta, \theta^{\prime}\right)=X_{t+\theta} X_{t+\theta^{\circ}}-R\left(\theta-\theta^{\prime}\right), \\
& \eta_{j}^{(T)}(t)=\int_{-b}^{0} \int_{-b}^{0} \psi_{j}(\theta) Z_{l}\left(\theta, \theta^{\prime}\right)\left[\alpha\left(\theta^{\prime}\right)-\alpha^{(T)}\left(\theta^{\prime}\right)\right] \mathrm{d} \theta \mathrm{d} \theta^{\prime},
\end{aligned}
$$

and

$$
\tilde{\eta}_{j}(t)=\int_{-b}^{0} \psi_{j}(\theta) X_{t+\theta} \mathrm{d} \theta
$$

Lemma 1. Assuming that $\int_{-\infty}^{\infty}|R(t)|^{2} \mathrm{~d} t<\infty$,

$$
E\left|f_{i j}^{(r)}-\delta_{i j}\right|^{2} \leqslant \frac{C}{T-t_{0}}
$$

for some constant $C$.

Proof.

$$
E\left|f_{i j}^{(T)}-\delta_{i j}\right|^{2}=\frac{1}{\left(T-t_{0}\right)^{2}} \int_{t_{0}}^{T} \int_{t_{0}}^{T} E \chi_{i j}(t) \chi_{i j}(s) \mathrm{d} t \mathrm{~d} s
$$

where

$$
\begin{aligned}
& \chi_{i j}(t)=\int_{-b}^{0} \int_{-h}^{0} \psi_{i}(\theta)\left[X_{t+\theta} X_{t+\theta^{\circ}}-R\left(\theta-\theta^{\prime}\right)\right] \psi_{j}\left(\theta^{\prime}\right) \mathrm{d} \theta \mathrm{d} \theta^{\prime}, \\
& E_{X_{i j}}(t) \chi_{i j}(s)= \iiint \int_{-b}^{0} \psi_{i}(\theta) \psi_{j}\left(\theta^{\prime}\right) \psi_{i}(\tau) \psi_{j}\left(\tau^{\prime}\right) \\
& \cdot\left[E X_{t+\theta} X_{t+\theta^{\prime}} X_{s+\tau} X_{s+\tau^{\prime}}-R\left(\theta-\theta^{\prime}\right) R\left(\tau-\tau^{\prime}\right)\right] \mathrm{d} \theta \mathrm{d} \theta^{\prime} \mathrm{d} \tau \mathrm{d} \tau^{\prime} .
\end{aligned}
$$

By the formula for the four products of Gaussian random variables,

$$
\begin{aligned}
E X_{t+\theta} X_{t+\theta^{\prime}} X_{s+\tau} X_{s+\tau^{\prime}}= & E X_{t+\theta} X_{t+\theta^{\prime}} E X_{s+\tau} X_{s+\tau^{\prime}}+E X_{t+\theta} X_{s+\tau} E X_{t+\theta^{\prime}} X_{s+\tau^{\prime}}+E X_{t+\theta} X_{s+\tau} E X_{t+\theta^{\prime}} X_{s+\tau} \\
= & R\left(\theta-\theta^{\prime}\right) R\left(\tau-\tau^{\prime}\right)+R(t-s+\theta-\tau) R\left(t-s+\theta^{\prime}-\tau^{\prime}\right) \\
& +R\left(t-s+\theta-\tau^{\prime}\right) R\left(t-s+\theta^{\prime}-\tau\right) .
\end{aligned}
$$

Therefore,

$$
\begin{aligned}
E\left|f_{i j}^{(T)}-\delta_{i j}\right|^{2}= & \frac{1}{T-t_{0}} \iiint_{-b}^{0} \psi_{i}(\theta) \psi_{j}\left(\theta^{\prime}\right) \psi_{i}(\tau) \psi_{j}\left(\tau^{\prime}\right) \\
& \cdot\left(\frac { 1 } { T - t _ { 0 } } \int _ { t _ { 0 } } ^ { T } \int _ { t _ { 0 } } ^ { T } \left[R(t-s+\theta-\tau) R\left(t-s+\theta^{\prime}-\tau^{\prime}\right)\right.\right. \\
& \left.\left.+R\left(t-s+\theta-\tau^{\prime}\right) R\left(t-s+\theta^{\prime}-\tau\right) \mathrm{d} t \mathrm{~d} s\right]\right) \mathrm{d} \theta \mathrm{d} \theta^{\prime} \mathrm{d} \tau \mathrm{d} \tau^{\prime}
\end{aligned}
$$

Now

$$
\begin{aligned}
& \frac{1}{T-t_{0}} \int_{t_{0}}^{T} \int_{t_{0}}^{T} R(t-s+\theta-\tau) R\left(t-s+\theta^{\prime}-\tau^{\prime}\right) \mathrm{d} t \mathrm{~d} s \\
& \quad=\int_{T-t_{0}}^{T-t_{0}} R(t+\theta-\tau) R\left(t+\theta^{\prime}-\tau^{\prime}\right)\left(1-\frac{t_{0}}{T}\right) \mathrm{d} t \\
& \leqslant \int_{-\infty}^{\infty}\left|R(t+\theta-\tau) \| R\left(t+\theta^{\prime}-\tau^{\prime}\right)\right| \mathrm{d} t \leqslant \int_{-\infty}^{\infty}|R(t)|^{2} \mathrm{~d} t .
\end{aligned}
$$

342 
A similar inequality holds for the other term and the results follows under the assumption that $\int_{-\infty}^{\infty}|R(t)|^{2} \mathrm{~d} t<\infty$.

Lemma 2. Under the same assumption as in Lemma 1,

$$
E\left\{\left|h^{(T)}\right|_{j}^{2}\right\} \leqslant\left[1+\left(\int_{-b}^{0} \psi_{j}(\theta)^{2} \mathrm{~d} \theta \int_{-b}^{0}\left[\alpha(\theta)-\alpha^{(T)}(\theta)\right]^{2} \mathrm{~d} \theta \int_{-\infty}^{\infty}|R(t)|^{2} \mathrm{~d} t\right)^{1 / 2}\right]^{2} /\left(T-t_{0}\right) .
$$

Proof. From (3.7), we get

$$
\begin{aligned}
& \left(E\left\{\left|h_{j}^{(T)}\right|^{2}\right\}\right)^{1 / 2} \leqslant\left\{E \frac{1}{\left(T-t_{0}\right)^{2}}\left(\int_{t_{0}}^{T} \eta_{j}^{(T)}(t) \mathrm{d} t\right)^{2}\right\}^{1 / 2}+\left\{E \frac{1}{\left(T-t_{0}\right)^{2}}\left(\int_{t_{0}}^{T} \tilde{\eta}_{j}(t) \mathrm{d} W_{t}\right)^{2}\right\}^{1 / 2}, \\
& E\left(\int_{t_{0}}^{T} \eta_{j}^{(T)}(t) \mathrm{d} t\right)^{2}=\int_{t_{0}}^{T} \int_{t_{0}}^{T} E \mu_{j}(t) \mu_{j}(s) \mathrm{d} t \mathrm{~d} s,
\end{aligned}
$$

where

$$
\begin{aligned}
E \mu_{j}(t) \mu_{j}(s)= & E \iiint \int_{-b}^{0} \psi_{j}(\theta) \psi_{j}(\tau)\left[\alpha\left(\theta^{\prime}\right)-\alpha^{(T)}\left(\theta^{\prime}\right)\right] \\
& \cdot\left[\alpha\left(\tau^{\prime}\right)-\alpha^{(T)}\left(\tau^{\prime}\right)\right] Z_{r}\left(\theta, \theta^{\prime}\right) Z_{s}\left(\tau, \tau^{\prime}\right) \mathrm{d} \theta \mathrm{d} \theta^{\prime} \mathrm{d} \tau \mathrm{d} \tau^{\prime} \\
= & \iiint_{-b}^{0} \psi_{j}(\theta) \psi_{j}(\tau)\left[\alpha\left(\theta^{\prime}\right)-\alpha^{(T)}\left(\theta^{\prime}\right)\right]\left[\alpha\left(\tau^{\prime}\right)-\alpha^{(T)}\left(\tau^{\prime}\right)\right] \\
& \cdot\left[R(t-s+\theta-\tau) R\left(t-s+\theta^{\prime}-\tau^{\prime}\right)+R\left(t-s+\theta-\tau^{\prime}\right) R\left(t-s+\theta^{\prime}-\tau\right)\right] \mathrm{d} \theta \mathrm{d} \theta^{\prime} \mathrm{d} \tau \mathrm{d} \tau^{\prime}
\end{aligned}
$$

Just as in the previous lemma,

$$
\frac{1}{T-t_{0}} \int_{t_{0}}^{T} \int_{t_{0}}^{T} R(t-s+\theta-\tau) R\left(t-s+\theta^{\prime}-\tau^{\prime}\right) \mathrm{d} t \mathrm{~d} s \leqslant \int_{-\infty}^{\infty}|R(t)|^{2} \mathrm{~d} t
$$

and we have, by the Schwartz inequality,

$$
\frac{1}{T-t_{0}} E\left(\int_{t_{0}}^{T} \eta_{j}^{(T)} \mathrm{d} t\right)^{2} \leqslant \int_{-b}^{0} \psi_{j}(\theta)^{2 \cdot} \mathrm{d} \theta \int_{-b}^{0}\left[\alpha(\theta)-\alpha^{(T)}(\theta)\right]^{2} \mathrm{~d} \theta \int_{-\infty}^{\infty}|R(t)|^{2} \mathrm{~d} t .
$$

On the other hand,

$$
\begin{aligned}
\frac{1}{T-t_{0}} E\left(\int_{t_{0}}^{T} \tilde{\eta}_{j}(t) \mathrm{d} W\right)^{2} & =\frac{1}{T-t_{0}} \int_{t_{0}}^{T} E \tilde{\eta}_{j}(t)^{2} \mathrm{~d} t \\
& =\frac{1}{T-t_{0}} \int_{t_{0}}^{T}\left(\int_{-b}^{0} \int_{-b}^{0} \psi_{j}(\theta) R(\theta-\tau) \psi_{j}(\tau) \mathrm{d} \theta \mathrm{d} \tau\right) \mathrm{d} t=1,
\end{aligned}
$$

since $\left(\psi_{j}, \psi_{j}\right)_{R}=1$, and the lemma follows.

We are now in a position to prove our main result:

Theorem. Under the assumption that $n_{T}^{2} / T \rightarrow 0$ as $T \rightarrow \infty,\left\|\xi^{(T)}-\xi^{(T)}\right\| \rightarrow 0$ in probability as $T \rightarrow \infty$.

Proof. By $\|M\|$ we denote the operator norm of a matrix $M$; that is, $\|M\|=\sup \{\|M x\|,\|x\| \leqslant 1\}$. Fix a number $d, 0<d<1$. Let

$$
A_{T}=\left\{\omega\left\{\sum_{i=1}^{n_{T}} \sum_{j=1}^{n_{T}}\left(f_{i j}^{(T)}-\delta_{i j}\right)^{2}\right\}^{1 / 2} \leqslant d\right\}
$$


With $\overline{A_{r}}$ denoting the complement of $A_{T}$, it follows from Lemma 1 that

$$
P\left(\bar{A}_{T}\right) \leqslant \frac{E \sum_{i} \sum_{j}\left(f_{i j}-\delta_{i j}\right)^{2}}{d^{2}} \leqslant \frac{n_{T}^{2} \cdot C}{d^{2}\left(T-t_{0}\right)}<\varepsilon / 2
$$

for $T>T_{1}(\varepsilon)$, for any $\varepsilon>0$ arbitrarily small. On the set $A_{T}, F^{(T)}$ is invertible and from (3.6), $\xi^{(T)}-\xi^{(T)}=\left[F^{(r)}\right]^{-1} h^{(T)}$. Since $\sum_{i=1}^{n_{r}} \sum_{j=1}^{n_{T}}\left(f_{i j}^{(T)}-\delta_{i j}\right)^{2}<1$ on $A_{T}$, it follows from results in matrix theory (see [4]) that

$$
\left\|\xi^{(T)}-\xi^{(T)}\right\| \leqslant\left[1-\left\{\sum_{i=1}^{n} \sum_{j=1}^{n}\left(f_{i j}^{(r)}-\delta_{i j}\right)^{2}\right\}^{1 / 2}\right]^{-1}\left\|h^{(T)}\right\| \leqslant \frac{1}{\sqrt{1-d}}\left\|h^{(T)}\right\| \quad \text { on } A_{T} .
$$

Therefore, for any $\delta>0$,

$$
\begin{aligned}
P\left(\left\{\left\|\xi^{(T)}-\xi^{(T)}\right\|>\delta\right\} \cap A_{T}\right) & \leqslant P\left(\left\|h^{(r)}\right\|>\delta \sqrt{1-d}\right) \\
& \leqslant \frac{E\left\|h^{(T)}\right\|^{2}}{\delta^{2}(1-d)}=\frac{\sum_{j=1}^{n_{r}} E\left\{h_{j}^{(T)}\right\}^{2}}{\delta^{2}(1-d)}<\varepsilon / 2,
\end{aligned}
$$

for $T>T_{2}(\varepsilon)$, by Lemma 2. It follows then thai for $T>\max \left(T_{1}(\varepsilon), T_{2}(\varepsilon)\right)$,

$$
\begin{aligned}
P\left(\left\|\xi^{(T)}-\xi^{(T)}\right\|>\delta\right) & =P\left(\left\{\left\|\xi^{(T)}-\xi^{(T)}\right\|>\delta\right\} \cap A_{T}\right)+P\left(\left\{\left\|\hat{\xi}^{(T)}-\xi^{(T)}\right\|>\delta\right\} \cap \overline{A_{T}}\right) \\
& \leqslant P\left(\left\{\left\|\xi^{(T)}-\xi^{(T)}\right\|>\delta\right\} \cap A_{T}\right)+P\left(\overline{A_{T}}\right)<\varepsilon,
\end{aligned}
$$

proving the desired result.

Corollary 1. If $n_{T} \rightarrow \infty$ and $n_{T}^{2} / T \rightarrow 0$ as $T \rightarrow \infty$, then

$$
\lim _{T \rightarrow \infty} \int_{t_{0}}^{T}\left[\hat{\alpha}^{(T)}(\theta)-\alpha(\theta)\right] R\left(\theta-\theta^{\prime}\right)\left[\hat{\alpha}^{(T)}\left(\theta^{\prime}\right)-\alpha\left(\theta^{\prime}\right)\right] \mathrm{d} \theta \mathrm{d} \theta^{\prime}=0
$$

in probability.

Proof. We write $\|f\|_{R}^{2}$ for $(f, f)_{R}$ and $\|f\|^{2}$ for $(f, f)$. The expression above can be written as

$$
\begin{aligned}
\lim _{T \rightarrow \infty}\left\|\hat{\alpha}^{(T)}(\theta)-\alpha(\theta)\right\|_{R}^{2} & =\lim _{T \rightarrow \infty}\left\|\hat{\alpha}^{(T)}(\theta)-\alpha^{(T)}(\theta)\right\|_{R}^{2}+\lim _{T \rightarrow \infty}\left\|\alpha^{(T)}(\theta)-\alpha(\theta)\right\|_{R}^{2} \\
& \leqslant \lim _{T \rightarrow \infty}\left\|\xi^{(T)}-\xi^{(T)}\right\|^{2}+\lim _{n_{T} \rightarrow \infty}\left\|\alpha^{(T)}(\theta)-\alpha(\theta)\right\|^{2} .
\end{aligned}
$$

Since $U_{n \geq 1} V_{n}$ is dense in $L_{2}[-b, 0]$, the result follows from the preceding theorem.

Corollary 2. Suppose that the operator $\mathscr{R}$ on $L_{2}[-b, 0]$ defined by

$$
\mathscr{R} f=g, \quad g(\theta)=\int_{-b}^{0} R\left(\theta-\theta^{\prime}\right) f\left(\theta^{\prime}\right) \mathrm{d} \theta^{\prime}, \quad-b \leqslant \theta \leqslant 0,
$$

is coercive, that is $(f, f) \geqslant \kappa\|f\|^{2}$ for some $\kappa>0$. Then, under the conditions of Corollary 1 ,

$$
\lim _{T \rightarrow \infty} \int_{t_{0}}^{T}\left|\hat{\alpha}^{(T)}(\theta)-\alpha(\theta)\right|^{2} \mathrm{~d} \theta=0
$$

in probability.

Proof. Obvious. 


\section{Conclusion}

Corollary 1 gives the natural consistency result for our problem. However, it is not totally satisfactory, since the function $R$ involved in the inner product contains the unknown parameter $\alpha(\cdot)$. What we ideally want is the result given in Corollary 2. It is, therefore, of interest to determine a sufficient condition ensuring coercivity of the operator $\mathscr{R}$.

It is easy to extend the results to the vector case and also to the more general hierarchical model, where both point and distributed delays occur.

\section{References}

[1] M. Polis, The distributed system parameter identification problem: a survey of recent results, Proceedings of the 3rd IFAC Symposium on Control of Distributed Parameter Systems (Preprints), Toulouse, France (1982).

[2] Ju. A. Rozanov, Infinite Dimensional Gaussian Distributions (American Mathematical Society, Providence, RI, 1971) (translation of the Russian original).

[3] V. Grenander, Abstract Inference (John Wiley, New York, 1981).

[4] H.T. Nguyen and T.D. Pham, Identification of nonstationary diffusion model by the method of sieves, SIAM J. Control Optim. 20 (1982) 603-611.

[5] K. Ito and M. Nisio, On stationary solutions of a stochastic differential equation, J. Math. Kyoto Univ. 4 (1964) 1-75.

[6] R.S. Lipster and A.M. Shiryayev, Statistics of Random Processes, I (Springer, New York, 1977). 